\title{
SUFFICIENT CONDITIONS FOR A WELL-BEHAVED KUROSH-AMITSUR RADICAL THEORY
}

\author{
by STEFAN VELDSMAN
}

(Received 4th December 1987)

\section{Introduction and preliminaries}

Kurosh-Amitsur radical theories have been developed for various algebraic structures. Whenever the notion of a normal substructure is not transitive, this causes quite some problems in obtaining satisfactory general results. Some of the more important questions concerning the general theory of radicals are whether semisimple classes are hereditary, do radical classes satisfy the ADS-property, can semisimple classes be characterized by closure conditions (e.g., is semisimple=coradical), is Sands' Theorem valid and lastly, does the lower radical construction terminate. For associative and alternative rings, all these questions have positive answers. The method of proof is the same in both cases. In [15], Puczylowski used the results of Terlikowska-Oslowska [18, 19] and hinted at a condition which is crucial in obtaining the positive answers to the above questions.

Our aim here is to make this more precise* and we provide sufficient conditions on a universal class of $\Omega$-groups to ensure positive answers to the above questions. We also give necessary conditions on a universal class for the semisimple classes to be hereditary and radical classes to have the ADS-property.

Although our results are presented in a universal class of multi-operator groups, it seems plausible that most of these can be extended to other types of universal classes or categories.

A class of $\Omega$-groups is a universal class if it is closed under homomorphic images and ideals. Ideals will be denoted by $\triangle$ and accessible $\Omega$-subgroups by $\triangle \triangle(D$ is an accessible $\Omega$-subgroup of $A$ if there exists a finite chain $D=$ $\left.D_{1} \triangle D_{2} \Delta \cdots \triangle D_{n-1} \triangle D_{n}=A\right)$. For definitions and characterizations of radical and semisimple classes, Van Leeuwen and Wiegandt $[20,21]$ can be consulted. We, however, recall the following: $A$ radical class $\mathscr{R}$ has the $A D S$-property if $\mathscr{R}(I) \triangle A$ for all $I \triangle A$ and is ideal-hereditary if $\mathscr{R}(I)=\mathscr{R}(A) \cap I$ for all $I \triangle A$. A class $\mathscr{M}$ is a semisimple class iff $\mathscr{M}$ is regular $(0 \neq I \triangle A \in \mathscr{M}$ implies $0 \neq I / K \in \mathscr{M}$ for some $K \triangle I)$, coinductive (if $I_{1} \supseteq \cdots \supseteq I_{\alpha} \supseteq \cdots$ is a descending chain, where $I_{\alpha} \triangle A$ with $A / I_{\alpha} \in \mathscr{M}$ for all $\alpha$, then

\footnotetext{
*While completing this manuscript, it came to my attention that E. R. Puczylowski (On general radical theory-manuscript) has obtained the same results, and in fact stronger conclusions, for the present $d_{3}(\mathscr{P})$-classes considered here in Section 3.
} 
$A / \cap I_{\alpha} \in \mathscr{M}$ ), closed under extensions ( $I \triangle A$ with both $I$ and $A / I$ in $\mathscr{M}$ implies $A \in \mathscr{M}$ and satisfies

(P) If $J \triangle I \triangle A$ and $J$ and $I$ are minimal w.r.t. $I / J \in \mathscr{M}$ and $A / I \in \mathscr{M}$, then $J \triangle A$.

For a class $\mathscr{M}$, two operators on $\mathscr{M}$ namely $\mathscr{U}$ and $\mathscr{S}$ are defined by

$\mathscr{U} \mathscr{M}:=\{A \mid A$ has no non-zero homomorphic image in $\mathscr{M}\}$ and

$\mathscr{S} \mathscr{M}:=\{A \mid A$ has no non-zero ideals which are in $\mathscr{M}\}$

If $\mathscr{M}$ is a regular (or equivalently, if $\mathscr{M} \subseteq \mathscr{S} \mathscr{U} \mathscr{M}$ ), then $\mathscr{U} \mathscr{M}$ is a radical class. $\mathscr{M}$ is a semisimple class iff $\mathscr{M}=\mathscr{S} \mathscr{U} \mathscr{M}$. $\mathscr{M}$ is a coradical class if it is hereditary (i.e. I $\triangle A \in \mathscr{M}$ implies $I \in \mathscr{M}$ ), coinductive and closed under extensions. For associative rings (cf. Sands [16]) and alternative rings (cf. Anderson and Wiegandt [4]), the following results is known as Sands' Theorem: $\mathscr{M}$ is a semisimple class iff $\mathscr{M}$ is regular, coinductive and closed under extensions.

We also need the lower radical construction (due to Kurosh [9] and streamlined by Suliński, Anderson and Divinsky [17]) in a universal class $\mathscr{W}$. Let $\mathscr{M} \subseteq \mathscr{W}$ and define classes $\mathscr{M}_{\alpha}$ by transfinite induction by: Let $\mathscr{M}_{1}$ be the homomorphic closure of $\mathscr{M}$. If $\mathscr{M}_{\beta}$ has been defined for all ordinals $\beta, \beta<\alpha$, let $\mathscr{M}_{\alpha}:=\{A \in \mathscr{W} \mid$ every non-zero homomorphic image of $A$ contains a non-zero ideal $I$ with $I \in \mathscr{M}_{\gamma}$ for some $\left.\gamma<\alpha\right\}$. Then $\mathscr{L} \mathscr{M}:=\bigcup_{\alpha} \mathscr{M}_{\alpha}$ is a radical class; in fact the smallest radical class which contains $\mathscr{M}$ and it is called the lower radical class determined by $\mathscr{M}$. It is well-known that if $\alpha<\beta$, then $\mathscr{M}_{\alpha} \subseteq \mathscr{M}_{\beta}$; each $\mathscr{M}_{\alpha}$ is homomorphically closed and if $\mathscr{M}$ is hereditary, so is each class $\mathscr{M}_{\alpha}$ and consequently also $\mathscr{L} \mathscr{M}$. Furthermore, if $0 \neq A \in \mathscr{L} \mathscr{M}$, then there is an accessible $\Omega$-subgroup $C \triangle \triangle A$ with $0 \neq C \in \mathscr{M}_{1}$. Quite often, we will be considering the lower radical construction determined by a single $\Omega$-group $A$; the steps in the construction of $\mathscr{L}\{A\}$ are then denoted by $\{A\}_{1},\{A\}_{2}, \ldots,\{A\}_{a}, \ldots$

Finally, as is usual when dealing with matters radical theoretical, any chosen subclass of a universal class will be assumed by be abstract, i.e. containing the one element $\Omega$ group and closed under isomorphic copies.

\section{General theory}

Let $\mathscr{W}$ be a universal class of $\Omega$-groups. Let $\mathscr{A} \subseteq \mathscr{W}$ be a fixed subclass with $\mathscr{A} \backslash\{0\} \neq \phi$. Motivated by Terlikowska-Oslowska [18], Puczylowski [15] and Krempa [8] we define:

Definitions 2.1. Let $J \triangle I \in \mathscr{W} . J$ is a $d_{i}(\mathscr{A})$-ideal of $I, i \in\{0,1,2,3,4\}$ if there does not exist a surjective homomorphism $\delta: B \rightarrow C / J \neq 0$ with:

for $i=0: B \triangle \triangle J, C=C_{1} \triangle C_{2} \triangle \cdots \triangle C_{n-1} \triangle C_{n}=I$ and $C_{n-1} / J \in \mathscr{A}$.

for $i=1: B=J, C=C_{1} \Delta C_{2} \triangle \cdots \Delta C_{n-1} \Delta C_{n}=I$ and $C_{n-1} / J \in \mathscr{A}$.

for $i=2: B=J, C \triangle I$ and $C / J \in \mathscr{A}$. 
for $i=3: B=J, C \triangle I, C / J \in \mathscr{A}$ and ker $\delta \triangle I$.

for $i=4$ : As for $i=3$ and if $K \triangle I$ with $K \subseteq J$, then $\pi^{-1}(\delta(K)) \triangle I$ where $\pi: C \rightarrow C / J$ is the canonical homomorphism.

$\mathscr{W}$ is a $d_{i}(\mathscr{A})$-class if $J \triangle I \triangle A \in \mathscr{W}$ and $J$ a $d_{i}(\mathscr{A})$-ideal of $I$ implies $J \triangle A$.

Clearly, for $i=1,2,3,4$, every $d_{i-1}(\mathscr{A})$-ideal is a $d_{i}(\mathscr{A})$-ideal; hence every $d_{i}(\mathscr{A})$-class is a $d_{i-1}(\mathscr{A})$-class. If $\mathscr{B} \subseteq \mathscr{A}$, then every $d_{i}(\mathscr{A})$-ideal is a $d_{i}(\mathscr{B})$-ideal; hence every $d_{i}(\mathscr{B})$-class is a $d_{i}(\mathscr{A})$-class. If $\mathscr{A}=\mathscr{W}, d_{i}(\mathscr{A})$-ideals $\left(d_{i}(\mathscr{A})\right.$-classes) will just be called $d_{i}$-ideals $\left(d_{i}\right.$-classes).

In the universal class of all rings, let $A$ be a simple ring without an identity and let $D(A)$ be the Dorroh extension of $A$. Then $D(A)$ is a $d_{1}$-ideal of $A \oplus D(A)$ but not a $d_{0}$-ideal. In [10], Leavitt and Armendariz gave an example of a (non-associative) ring $K$ with order 8 and with a chain $0 \neq J \Delta I \triangle K$ where $I$ is the only proper ideal of $K$ and $J$ is the only proper ideal of $I . I$ and $K$ have no identity and $J$ is isomorphic to the two element field. If $D(K)$ is the Dorroh extension of $K$, then $D(K)$ is a $d_{2}$-ideal of $K \oplus D(K)$ but not a $d_{1}$-ideal. In the same paper, Leavitt and Armendariz also gave an example of a (non-associative) ring $R$ with order 8 and with a chain $0 \neq J \triangle I \triangle R$ where $I$ is the only proper ideal of $R, J$ is the only proper ideal of $I$ and $R^{2}=R, I^{2}=I$ and $J^{2}=0$. Then $I$ is a $d_{3}$-ideal of $R$, but not a $d_{2}$-ideal.

Let $I \triangle A \in \mathscr{W}$. If $I \in \mathscr{U} \mathscr{A}$ then $I$ is a $d_{2}(\mathscr{A})$-ideal of $A$ and if $A / I \in \mathscr{S} \mathscr{A}$, then $I$ is a $d_{1}(\mathscr{A})$-ideal of $A$. If $\mathscr{A}$ is hereditary and $I \in \mathscr{U} \mathscr{A}$, then $I$ is a $d_{1}(\mathscr{A})$-ideal of $A$ and if, in addition, also $\mathscr{U} \mathscr{A}$ is hereditary, then $I$ is a $d_{0}(\mathscr{A})$-ideal of $A$. Moreover, the next two results can easily be verified:

Proposition 2.2. Let $\mathscr{M} \subseteq \mathscr{W}$ be hereditary and closed under extensions. If I $\triangle A \in \mathscr{W}$ and $I$ is minimal with respect to $0 \neq A / I \in \mathscr{M}$, then $I$ is a $d_{3}$-ideal of $A$.

Proposition 2.3. Let $\mathscr{R} \subseteq \mathscr{W}$ be a radical class.

(1) $\mathscr{R}(A)$ is a $d_{2}(\mathscr{A})$-ideal of $A$ for all $A \in \mathscr{W}$.

(2) If $\mathscr{S} \mathscr{R}$ is hereditary, then $\mathscr{R}(A)$ is a $d_{1}(\mathscr{A})$-ideal of $A$ for all $A \in \mathscr{W}$.

(3) If $\mathscr{R}$ is ideal-hereditary, then $\mathscr{R}(A)$ is a $d_{0}(\mathscr{A})$-ideal of $A$ for all $A \in \mathscr{W}$.

Corollary 2.4. Let $\mathscr{W}$ be a $d_{2}(\mathscr{A})$-class. Then every radical class in $\mathscr{W}$ satisfies the ADS-property; consequently the semisimple classes in $\mathscr{W}$ are hereditary.

$d_{1}$ - and $d_{0}$-ideals arise as the radicals for certain radical classes, for we have:

Proposition 2.5. Let $I \triangle A \in \mathscr{W}$.

(1) If I is a $d_{1}$-ideal of $A$, then there exists a radical class $\mathscr{R}$ with $\mathscr{R}(A)=I$.

(2) If I is a $d_{0}$-ideal of $A$, then there exists a hereditary radical class $\mathscr{R}$ with $\mathscr{R}(A)=I$.

Proof. (1) Let $\mathscr{R}:=\mathscr{L}\{I\}$. Then $I \in \mathscr{R}$, hence $I \subseteq \mathscr{R}(A)$. If $I \neq \mathscr{R}(A)$, then $\mathscr{R}(A) / I \in \mathscr{R}$ 
implies the existence of a non-zero accessible $\Omega$-subgroup $C / I$ of $\mathscr{R}(A) / I$ which is a homomorphic image of $I$. But this contradicts the fact that $I$ is a $d_{1}$-ideal of $A$.

(2) In this case, let $\mathscr{R}=\mathscr{L}\{K \mid K$ is an accessible $\Omega$-subgroup of $I\}$. Since the class which generates $\mathscr{R}$ is hereditary, so is $\mathscr{R}$ and we can proceed as in (1).

Let us remark that if $\mathscr{W}$ is such that all semisimple classes in $\mathscr{W}$ are hereditary, then the conditions in both (1) and (2) of Proposition 2.5 are necessary and sufficient. In a sense, the next two results motivate our approach in considering the classes presently under discussion.

Proposition 2.6. Let $\mathscr{W}$ be a universal class in which every semisimple class is hereditary. Then $\mathscr{W}$ is a $d_{0}$-class.

Proof. If $\mathscr{W}$ is not a $d_{0}$-class, there is a chain $J \Delta I \triangle A \in \mathscr{W}$ with $J$ a $d_{0}$-ideal in $I$ but not an ideal in $A$. As in the proof of Proposition 2.5(2), if $\mathscr{R}:=\mathscr{L}\{K \mid K$ is an accessible $\Omega$-subgroup of $J\}$, it follows that $\mathscr{R}$ is a hereditary radical class with $\mathscr{R}(I)=J$. By our assumption on $\mathscr{W}, \mathscr{R}$ is in fact ideal-hereditary. But then, as is well-known, $\bar{J} \in \mathscr{R}$ where $\bar{J}$ is the ideal in $A$ generated by $J$. Hence $\bar{J} \subseteq \mathscr{R}(I)=J$ which contradicts the fact that $J$ is not an ideal in $A$.

Proposition 2.7. Let $\mathscr{W}$ be a universal class in which every radical class has the ADS-property. Then $\mathscr{W}$ is a $d_{1}$-class.

Proof. Consider the chain $J \triangle I \triangle A \in \mathscr{W}$ where $J$ is a $d_{1}$-ideal of $I$. From Proposition 2.5(1) we know that there is a radical class $\mathscr{R}$ with $J=\mathscr{R}(I)$ and $J \triangle A$ follows from our assumption on $\mathscr{W}$.

It is well-known (trivially) that every radical class which has the ADS-property has a hereditary semisimple class. Moreover, in all the universal classes where it is known that the semisimple classes are hereditary, it is precisely because all the radical classes have the ADS-property. Although the ADS-property for a radical class is not necessary for the corresponding semisimple class to be hereditary (cf. [22]), no universal class is known where all the semisimple classes are hereditary but not every radical class has the ADS-property. The next result, which is also a partial converse to the last proposition, provides some information.

Proposition 2.8. Let $\mathscr{W}$ be a universal class. Then every radical class in $\mathscr{W}$ has the ADS-property if and only if every semisimple class in $\mathscr{W}$ is hereditary and $\mathscr{W}$ is a $d_{1}$-class.

The proof is obvious in view of Propositions 2.7 and 2.3(2). We conclude this section with two properties that are quite useful in the study of general radical theory; the proofs are straightforward. 
Proposition 2.9. Let $J \triangle I \triangle A \in \mathscr{W}$. If $\mathscr{W}$ is a $d_{1}(\mathscr{A})$-class and $I / J \in \mathscr{S} \mathscr{A}$, then $J \triangle A$. If $\mathscr{W}$ is a $d_{1}(\mathscr{A})$-class and $\mathscr{A}$ is hereditary or if $\mathscr{W}$ is a $d_{2}(\mathscr{A})$-class, then $J \in \mathscr{U} \mathscr{A}$ implies $J \triangle A$.

\section{Characterizations of semisimple classes}

Theorem 3.1. Let $\mathscr{W}$ be a $d_{3}$-class. Then $\mathscr{M} \subseteq \mathscr{W}$ is a semisimple class if and only if it is a coradical class.

Proof. Let $\mathscr{M}$ be a coradical class. In order to show that $\mathscr{M}$ is a semisimple class, it is sufficient to show that $\mathscr{M}$ satisfies condition $(P)$. Consider the chain $J \triangle I \triangle A \in \mathscr{W}$ where $J$ and $I$ are minimal with respect to $I / J \in \mathscr{M}$ and $A / I \in \mathscr{M}$ respectively. From Proposition 2.2 it follows that $J$ is a $d_{3}$-ideal of $I$; hence $J \triangle A$ by the assumption on $\mathscr{W}$. The converse implication is clear from Corollary 2.4 and the well-known properties of semisimple classes.

Concerning the validity of Sands'-Theorem, we firstly have:

Theorem 3.2. Let $\mathscr{W}$ be a $d_{3}(\mathscr{A})$-class. Then $\mathscr{M} \subseteq \mathscr{W}$ is a semisimple class if and only if $\mathscr{M}$ is regular, coinductive, closed under extensions and satisfies:

$$
I \triangle A \in \mathscr{M} \text { and } I \in \mathscr{A} \text { implies } I \in \mathscr{M} \text {. }
$$

Proof. Suppose $\mathscr{M}$ satisfies the mentioned conditions. Since $\mathscr{M}$ is regular, $\mathscr{M} \subseteq$ $\mathscr{S} \mathscr{U} \mathscr{M}$. We show that $\mathscr{M}=\mathscr{S} \mathscr{U} \mathscr{M}$ from which the result follows. Let $0 \neq A \in \mathscr{S} \mathscr{U} \mathscr{M}$. Then there is an ideal $I \triangle A$ such that $0 \neq A / I \in \mathscr{M}$. Since $\mathscr{M}$ is coinductive, we can choose $I$ minimal with respect to this property. If $I=0$, we are done. Suppose thus $0 \neq I \triangle A \in \mathscr{S} \mathscr{U} \mathscr{M}$. Once again, we can find an ideal $J \triangle I$ with $0 \neq I / J \in \mathscr{M}$ where $J$ is minimal w.r.t. this property. We now show that $J$ is a $d_{3}(\mathscr{A})$-ideal of $I$. If not, there exists a surjective homomorphism $\delta: J \rightarrow C / J$ where $C \triangle I, C / J \in \mathscr{A} \backslash\{0\}$ and $\operatorname{ker} \delta \triangle I$. Then $J / \operatorname{ker} \delta \cong C / J \triangle I / J \in \mathscr{M}$ and from (*) we infer that $C / J$ and consequently $J / \operatorname{Ker} \delta$ is in $\mathscr{M}$. Since $J / \operatorname{ker} \delta \triangle I / \operatorname{ker} \delta$ and

$$
\frac{I / \operatorname{ker} \delta}{J / \operatorname{ker} \delta} \cong \frac{I}{J} \in \mathscr{M}
$$

the extension closedness of $\mathscr{M}$ yields $I / \operatorname{ker} \delta \in \mathscr{M}$ which contradicts the choice of $J$. Thus $J$ is a $d_{3}(\mathscr{A})$-ideal of $I$ and then also an ideal in $A$. Once again, since $\mathscr{M}$ is closed under extensions and from

$$
\frac{A / J}{I / J} \cong \frac{A}{I} \in \mathscr{M}
$$


we conclude that $A / J \in \mathscr{M}$ which contradicts the choice of $I$. The converse implication is clear.

For the validity of Sands' Theorem, one would like to know when (*) is a consequence of the other conditions imposed on $\mathscr{M}$. The remainder of this section is devoted to this problem. We start with two special cases.

Proposition 3.3. Suppose $\mathscr{A}$ is homomorphically closed and for all $J \triangle I \triangle A \in \mathscr{W}$ with $I / J \in \mathscr{S} \mathscr{A}, J \triangle A$ holds. Then $\mathscr{M} \subseteq \mathscr{W}$ is the semisimple class of a radical class $\mathscr{R}$ for which $\mathscr{A} \subseteq \mathscr{R}$ if and only if $\mathscr{M}$ is regular, coinductive, closed under extensions, $\mathscr{M} \cap \mathscr{A}=0$ and $\mathscr{R}=\mathscr{U} \mathscr{M}$. Furthermore, the radical class $\mathscr{R}$ has the ADS-property.

Proof. Assume $\mathscr{M}$ satisfies the mentioned conditions and let $\mathscr{R}:=\mathscr{U} \mathscr{M}$. Since $\mathscr{M}$ is regular, $\mathscr{R}$ is a radical class and if $A \in \mathscr{A}$, then $A / \mathscr{R}(A) \in \mathscr{S} \mathscr{R} \cap \mathscr{A}$ since $\mathscr{A}$ is homomorphically closed. If $A / \mathscr{R}(A) \neq 0$, then $A / \mathscr{R}(A)$ has a non-zero homomorphic image in $\mathscr{M}$; consequently in $\mathscr{M} \cap \mathscr{A}$ which contradicts $\mathscr{M} \cap \mathscr{A}=0$. Hence $A=\mathscr{R}(A) \in \mathscr{R}$. To show that $\mathscr{S} \mathscr{R}=\mathscr{M}$, it is sufficient to show that $\mathscr{M}$ satisfies condition $(P)$. Consider thus the chain $J \triangle I \triangle A \in \mathscr{W}$ where $J$ and $I$ are minimal with respect of $I / J \in \mathscr{M}$ and $A / I \in \mathscr{M}$. As $\mathscr{A} \subseteq \mathscr{R}$, we have $I / J \in \mathscr{M} \subseteq \mathscr{S} \mathscr{U} \mathscr{M}=\mathscr{S} \mathscr{R} \subseteq \mathscr{S} \mathscr{A}$; hence by our assumption $J \triangle A$ follows. The converse is clear; so is the fact that $\mathscr{R}$ has the ADS-property since $I / \mathscr{R}(I) \in \mathscr{S} \mathscr{R} \subseteq \mathscr{S} \mathscr{A}$.

Corollary 3.4. Let $\mathscr{A}$ be homomorphically closed and suppose $\mathscr{W}$ is a $d_{1}(\mathscr{A})$-class. Then $\mathscr{M} \subseteq \mathscr{W}$ is the semisimple class of a radical class $\mathscr{R}$ for which $\mathscr{A} \subseteq \mathscr{R}$ if and only if $\mathscr{M}$ is regular, coinductive, closed under extensions, $\mathscr{M} \cap \mathscr{A}=0$ and $\mathscr{R}=\mathscr{U} \mathscr{M}$.

In view of Proposition 2.9, the hypothesis of Proposition 3.3 is satisfied from which the proof of the above corollary follows. The proof of the next result is immediate from Theorem 3.2.

Proposition 3.5. Let $\mathscr{A}$ be hereditary and suppose $\mathscr{W}$ is a $d_{3}(\mathscr{A})$-class. Then $\mathscr{M} \subseteq \mathscr{W}$ is the semisimple class of a radical class $\mathscr{R}$ for which $\mathscr{R} \subseteq \mathscr{U} \mathscr{A}$ iff $\mathscr{M}$ is regular, coinductive, closed under extensions, $\mathscr{A} \subseteq \mathscr{M}$ and $\mathscr{R}=\mathscr{U} \mathscr{M}$.

Let $\mathscr{B} \subseteq \mathscr{W}$ be a class with the property $J \triangle I \triangle A \in \mathscr{B}$ implies $J \triangle A$. In this case (as in all our other choices for $\mathscr{A}$ ), we tacitly assume that $\mathscr{B} \neq 0$ in $\mathscr{W}$ when considering $d_{i}(\mathscr{B})$-classes.

Theorem 3.6. Let $\mathscr{W}$ be a $d_{3}(\mathscr{B})$-class. Then $\mathscr{M} \subseteq \mathscr{W}$ is a semisimple class iff $\mathscr{M}$ is regular, coinductive and closed under extensions.

Proof. We only have to show that condition $(*)$ is a consequence of the other conditions imposed on $\mathscr{M}$ (cf. Theorem 3.2). Let $0 \neq I \triangle A \in \mathscr{M}$ with $I \in \mathscr{B}$. By the regularity and coinductiveness of $\mathscr{M}$, choose $J \triangle I$ minimal w.r.t. $0 \neq I / J \in \mathscr{M}$. Suppose 
$J \neq 0$. We distinguish two cases. If $J \triangle A$, then the regularity of $\mathscr{M}$ yields a non-zero homomorphic image of $J$ which is in $\mathscr{M}$. If $J$ is not an ideal in $A$, it is not a $d_{3}(\mathscr{B})$-ideal of $I$. Then there are ideals $K$ and $C$ of $I$ with $K \subseteq J \subseteq C$ and $J / K \cong C / J \in \mathscr{B} \backslash\{0\}$. Since $0 \neq C / J \triangle I / J \in \mathscr{M}$ and $\mathscr{M}$ is regular, $C / J$ and thus also $J$, has a non-zero homomorphic image which is in $\mathscr{M}$. Thus, in both cases we can find an ideal $B \triangle J$ with $0 \neq J / B \in \mathscr{M}$. Since $B \triangle J \triangle I \in \mathscr{B}, B \triangle I$ holds. From

$$
\frac{I / B}{J / B} \cong \frac{J}{J} \in \mathscr{M}
$$

and the fact that $\mathscr{M}$ is closed under extensions, $I / B \in \mathscr{M}$. But this contradicts the choice of $J$; hence $J=0$ and $I \in \mathscr{A}$ follows which proves the theorem.

For our next choices of $\mathscr{A}$, we have to fix some notation and recall some $\Omega$-group notions, mainly from Higgins [7]. Let $A \in \mathscr{W}$. By $A \Omega$ we denote the subset of $A$ defined by

$$
A \Omega:=\left\{\underline{a} \omega \mid \omega \in \Omega \text { and } \underline{a} \omega=a_{1} a_{2} \ldots a_{n} \omega \text { where } a_{i} \in A\right\} .
$$

$A$ is a trivial $\Omega$-group if $A \Omega=0$. Let $\mathscr{Z}$ be the class of all trivial $\Omega$-groups in $\mathscr{W}$. It is easy to verify that $\mathscr{Z}$ is homomorphically closed and hereditary. A radical class $\mathscr{R} \subseteq \mathscr{W}$ is hypersolvable if $\mathscr{Z} \subseteq \mathscr{R}$ and hypoidempotent of $\mathscr{R} \subseteq \mathscr{U} \mathscr{Z}$ (or equivantly, if $\mathscr{Z} \subseteq \mathscr{S} \mathscr{R}$ ). Propositions 3.4 and 3.5 give characterizations of the semisimple classes of hypersolvable and hypoidempotent radical classes in $d_{3}(\mathscr{Z})$-classes. For certain $d_{3}(\mathscr{L})$-classes, these results can be extended to arbitrary semisimple classes.

Convention. For the duration of this section, we assume that $\Omega \neq \phi$ and any $\omega \in \Omega$ has weight at least 2 .

We say that $\mathscr{W}$ is abelian if $A^{+}$is abelian for all $A \in \mathscr{W}$ where $A^{+}$is' the underlying group of $A$.

Theorem 3.7. Let $\mathscr{W}$ be an abelian $d_{3}(\mathscr{Z})$-class. Then $\mathscr{M} \subseteq \mathscr{W}$ is a semisimple class iff $\mathscr{M}$ is regular, coinductive and closed under extensions.

Since $\mathscr{W}$ is abelian, $\mathscr{Z} \subseteq \mathscr{B}$ and the result follows from Theorem 3.6.

For subsets $C$ and $D$ of $A \in \mathscr{W}$, the commutor group $[C, D]$ of $C$ and $D$ is the S-subgroup of $A$ consisting of all $f(\underline{c}, \underline{d})$ with $\underline{c} \in C$ and $\underline{d} \in D$ where $f(\underline{x}, \underline{y)}$ is a commutator word in $\underline{x}$ and $\underline{y}$. From Theorem 4.B in [7], it follows that if $C, D \triangle A$, then $[C, D]$ is the ideal of $C+D$ generated by $-c-d+c+d$ and $-\underline{c} \omega-\underline{d} \omega+(\underline{c}+\underline{d}) \omega$ for all $c, \underline{c} \in C, d, \underline{d} \in D$ and $\omega \in \Omega$. The lower central series

$$
A^{1} \supseteq A^{2} \supseteq A^{3} \supseteq
$$


and the derived series

$$
A^{(0)} \supseteq A^{(1)} \supseteq A^{(2)} \supseteq \ldots
$$

are defined inductively by

$$
A^{1}=A, A^{i}=\left[A^{i-1}, A\right] \text { for } i=2,3,4, \ldots
$$

and

$$
A^{(0)}=A, A^{(i)}=\left[A^{(i-1)}, A^{(i-1)}\right] \text { for } i=1,2,3, \ldots
$$

For each $i=1,2,3, \ldots, A^{i} \triangle A$ and $A^{(i)} \triangle A^{(i-1)}$.

$A$ is nilpotent (soluble; sometimes called solvable) if $A^{n}=0\left(A^{(n)}=0\right.$ respectively) for some $n \geqq 1$. Every nilpotent $\Omega$-group is soluble. For groups, associative rings, Lie rings, etc., these notions coincide with the usual. $A \in \mathscr{W}$ is abelian if $A^{2}=0$. This means $A^{+}$is abelian; the converse need not be true. If $\omega \in \Omega$, then $\omega$ is distributive on $A$ if

$$
a_{1} \ldots a_{i-1}\left(a_{i}+b\right) a_{i+1} \ldots a_{n} \omega=\underline{a} \omega+a_{1} \ldots a_{i-1} b a_{i+1} \ldots a_{n} \omega
$$

for all $b, \underline{a} \in A$ and $i=1,2, \ldots, n$.

$\omega$ is associative on $A$ if for all $a_{1}, a_{2}, \ldots, a_{2 n-1} \in A$,

$$
\begin{aligned}
& a_{1} a_{2} \ldots a_{i}\left(a_{i+1} \ldots a_{i+n} \omega\right) a_{i+n+1} \ldots a_{2 n-1} \omega \\
& \quad=a_{1} \ldots a_{i+1}\left(a_{i+2} \ldots a_{i+n+1} \omega\right) a_{i+n+2} \ldots a_{2 n-1} \omega
\end{aligned}
$$

for all $i=0,1,2, \ldots, n-2$.

$A$ is a distributive (associative) $\Omega$-group if $\omega$ is distributive (respectively associative) on $A$ for all $\omega \in \Omega$ and $\mathscr{W}$ is distributive (associative) if $A$ is distributive (respectively associative) for all $A \in \mathscr{W}$. If $A$ is a distributive $\Omega$-group, then $\underline{a} \omega=0$ if $a_{i}=0$ for some $i$ and also,

$$
-\underline{a} \omega=a_{1} \ldots a_{i-1}\left(-a_{i}\right) a_{i+1} \ldots a_{n} \omega
$$

for all $i$.

Lastly, $\mathscr{W}$ is a class of 0 -symmetric $\Omega$-groups if each $A \in \mathscr{W}$ is 0 -symmetric, i.e. for all $\omega \in \Omega$ and $\underline{a} \in A$, we have $\underline{a} \omega=0$ if $a_{i}=0$ for some $i$. Of course, if $\mathscr{W}$ is distributive, then $\mathscr{W}$ consists of 0 -symmetric $\Omega$-groups.

Proposition 3.8. Let $A$ be a 0-symmetric $\Omega$-group and let $k \geqq 2$. If $\omega \in \Omega, \underline{a} \in A$ and $a_{i} \in A^{k-1}$ for some $i$, then $\underline{a} \omega \in A^{k}$.

Proof. Let $\underline{x}=\left(0,0, \ldots, 0, a_{i}, 0, \ldots, 0\right)$ and let $\underline{y}=\left(a_{1}, \ldots, a_{i-1}, 0, a_{i+1}, \ldots, a_{n}\right)$. Then $\underline{x} \in A^{k-1}$ and $\underline{y} \in A$. Hence 


$$
\underline{a} \omega=-\underline{x} \omega-\underline{y} \omega+(\underline{x}+\underline{y}) \omega \in\left[A^{k-1}, A\right]=A^{k} .
$$

The following can easily be verified:

Proposition 3.9. Let $A$ be a 0 -symmetric $\Omega$-group. Then $A^{2}=0$ if and only if $A^{+}$is abelian and $A \Omega=0$.

Lemma 3.10. Let $\mathscr{M} \subseteq \mathscr{W}$ be coinductive and closed under extensions. Then $I \triangle A \in \mathscr{S} \mathscr{U} \mathscr{M}$ and $A^{2}=0$ implies $I \in \mathscr{M}$.

Proof. If $I=0$, we are done. Suppose $I \neq 0$. Then, from the definition of $\mathscr{S} \mathscr{U} \mathscr{M}$, there is an ideal $J \triangle I$ such that $0 \neq I / J \in \mathscr{M}$. Since $\mathscr{M}$ is coinductive, we can choose $J$ minimal w.r.t. this property. Since $A^{2}=0, J \triangle A$ holds. If $J \neq 0$, choose $K \triangle J$ minimal w.r.t. $0 \neq J / K \in \mathscr{M}$. From $I^{2}=0$, we have $K \triangle I$ and because $\mathscr{M}$ is closed under extension and $(I / K) /(J / K) \cong I / J \in \mathscr{M}, I / K \in \mathscr{M}$. This contradicts the choice of $J$; hence $J=0$ and $I \in \mathscr{M}$.

Lemma 3.11. Let $\mathscr{W}$ be a $d_{3}$-class. If $\mathscr{M} \subseteq \mathscr{W}$ is regular, coinductive and closed under extensions, then $I \triangle A \in \mathscr{S} \mathscr{U} \mathscr{M}$ and $I^{2}=0$ implies $I \in \mathscr{M}$.

Proof. Suppose $I \neq 0$ and choose $J \triangle I$ minimal w.r.t. $0 \neq I / J \in \mathscr{M}$. Then $J \triangle A$; if not there are ideals $K, C \triangle I$ with $K \subseteq J \subseteq C$ and $0 \neq J / K \cong C / J$. Since $I^{2}=0$, so is $I / J$ and from Lemma $3.10, C / J$ and thus also $J / K$ is in $\mathscr{M}$. From $(I / K) /(J / K) \cong I / J \in \mathscr{M}$ we have $I / K \in \mathscr{M}$ which contradicts the choice of $J$. Thus, $J \triangle A$. If $J \neq 0$, choose $B \triangle J$ minimal w.r.t. $0 \neq J / B \in \mathscr{M}$. Since $J^{2}=0$, the same reasoning as above yields $B \triangle I$. Then $I / B \in \mathscr{M}$ which contradicts the choice of $J$. Hence $J=0$ and $I \in \mathscr{M}$ holds.

Let $\mathscr{T}:=\left\{A \in \mathscr{W} \mid A^{2}=0\right\}$. If $\mathscr{W}$ is abelian, then $\mathscr{Z} \subseteq \mathscr{T}$ and, if in addition, $\mathscr{W}$ is also 0 -symmetric, $\mathscr{Z}=\mathscr{T}$. However, in general the equality need not hold.

Combining Lemma 3.11 and Theorem 3.2, we immediately have:

Theorem 3.12. Let $\mathscr{W}$ be a $d_{3}(\mathscr{T})$-class. Then $\mathscr{M} \subseteq \mathscr{W}$ is a semisimple class if and only if $\mathscr{M}$ is regular, coinductive and closed under extensions.

Let us mention that this result also follows from Theorem 3.6 since $\mathscr{T} \subseteq \mathscr{B}$.

Let $\mathcal{N}:=\left\{A \in \mathscr{W} \mid A^{n}=0\right.$ for some $\left.n \geqq 1\right\}$. In order to extend Theorem 3.12 to the more general $d_{3}(\mathscr{N})$-classes, it appears that we have to impose some restrictions on the operators in $\Omega$. Firstly we need a certain subset $A^{*}$ of $A \in \mathscr{W}$ which generalizes the concept of an annihilator:

$$
\begin{gathered}
A^{*}:=\left\{a \in A \mid \text { for all } \omega \in \Omega \text { and } \underline{b} \in A,-\underline{b} \omega+b_{1} \ldots b_{i-1}\left(a+b_{i}\right) b_{i+1} \ldots\right. \\
\left.\qquad b_{n} \omega=0 \text { for all } i=1,2, \ldots, n\right\} .
\end{gathered}
$$

Proposition 3.13. Let $A$ be a distributive $\Omega$-group. Then $A^{*} \triangle A$; in fact for any $\Omega$-subgroup $B$ of $A$ for which $B^{+}$is normal in $A^{+}$and $B \subseteq A^{*}, B \triangle A$ holds. 
Proof. Since $A$ is distributive, $A^{*}$ simplifies to

$$
A^{*}:=\left\{a \in A \mid \text { for all } \omega \in \Omega \underline{b} \in A, b_{1} b_{2} \ldots b_{i-1} a b_{i+1} \ldots b_{n} \omega=0 \text { for all } i=1,2, \ldots, n\right\}
$$

Let $a, b \in A^{*}, \omega \in \Omega, \underline{x}, x \in A$. For any $i \in\{1,2, \ldots, n\}$ we have

$$
\begin{gathered}
x_{1} \ldots x_{i-1}(a-b) x_{i+1} \ldots x_{n} \omega=0 \\
x_{1} \ldots x_{i-1}(x+a-x) x_{i+1} \ldots x_{n} \omega=0
\end{gathered}
$$

and

$$
x_{1} \ldots x_{i-1} a x_{i+1} \ldots x_{n} \omega=0
$$

Hence $A^{*} \triangle A$.

Proposition 3.14. Let $A$ be a distributive $\Omega$-group. Then $A^{k}=0$ implies $A^{k-1} \subseteq A^{*}$.

Proof (by induction on $k$ ). If $k=2$, then $A \Omega=0$ (Proposition 3.9). Thus $A^{1}=A \subseteq A^{*}$. Suppose $A^{k+1}=0, k \geqq 2$. In view of Proposition 3.13 , it is sufficient to verify that the generators of $A^{k}=\left[\bar{A}^{k-1}, A\right]$, namely

$$
-a-b+a+b, a \in A^{k-1}, b \in A
$$

and

$$
-\underline{a} \omega-\underline{b} \omega+(\underline{a}+\underline{b}) \omega, \omega \in \Omega, \underline{a} \in A^{k-1}, b \in A
$$

are in $A^{*}$.

Let $\omega^{\prime} \in \Omega, \underline{x} \in A$. Then

$$
x_{1} \ldots x_{i-1}(-a-b+a+b) x_{i+1} \ldots x_{n} \omega^{\prime} \in A^{k+1}=0
$$

by Proposition 3.8 since $a \in A^{k-1}$ and $b \in A$ implies $-a-b+a+b \in\left[A^{k-1}, A\right]=A^{k}$. Hence $-a-b+a+b \in A^{*}$ for all $a \in A^{k-1}, b \in A$.

Secondly, consider

$$
z:=x_{1} \ldots x_{i-1}(-\underline{a} \omega-\underline{b} \omega+(a+\underline{b}) \omega) x_{i+1} \ldots x_{n} \omega^{\prime}
$$

Since $\underline{a} \in A^{k+1}$ and $\underline{b} \in A$, we have

$$
-\underline{a} \omega-\underline{b} \omega+(\underline{a}+\underline{b}) \omega \in\left[A^{k-1}, A\right]=A^{k} .
$$

Thus $z \in A^{k+1}=0$ from Proposition 3.8 and 


$$
-\underline{a} \omega-\underline{b} \omega+(\underline{a}+\underline{b}) \omega \in A^{*} \text { follows. }
$$

Our next four results are the $\Omega$-group variants of the corresponding ring-theoretic versions from Ánh, Loi and Wiegandt [5] and Ánh and Wiegandt [6] and are proved by using the same arguments.

Proposition 3.15. Let $\mathscr{M} \subseteq \mathscr{W}$ be closed under extensions and consider the chain

$$
I_{n} \triangle I_{n-1} \triangle \ldots \triangle I_{1} \triangle I_{0}=A \text { with } I_{n} \triangle A \in \mathscr{W} .
$$

If $I_{k} / I_{k+1} \in \mathscr{M}$ for all $k=0,1,2, \ldots, n-1$, then $A / I_{n} \in \mathscr{M}$.

Corollary 3.16 Let $\mathscr{M} \subseteq \mathscr{W}$ be closed under extensions. If $\mathscr{T} \subseteq \mathscr{M}$, then $\mathscr{M}$ contains all soluble (and hence all nilpotent) $\Omega$-groups from $\mathscr{W}$.

Proposition 3.17 Let $\mathscr{M} \subseteq \mathscr{W}$ be regular and closed under extensions. If $I_{n} \triangle I_{n-1} \triangle \cdots \Delta I_{1} \Delta I_{0}=A \in \mathscr{W}$ and $I_{k} / I_{k+1} \in \mathscr{M}$ for all $k=0,1,2, \ldots, n-1$; then $\mathscr{R}(A) \subseteq \mathscr{R}\left(I_{n}\right)$ where $\mathscr{R}:=\mathscr{U} \mathscr{M}$.

Proposition 3.18. Let $\mathscr{W}$ be abelian and distributive. Let $\mathscr{M} \subseteq \mathscr{W}$ be regular, coinductive, closed under extensions and assume $\mathscr{S} \mathscr{U} \mathscr{M}$ is hereditary. Then $\mathscr{A} \in \mathscr{S} \mathscr{U} \mathscr{M}$ and $A$ nilpotent implies $A \in \mathscr{M}$.

Proof (by induction on $k$, the degree of nilpotency of $A$ ). If $A^{2}=0$, the result follows from Lemma 3.10. Assume the result holds for $k-1 \geqq 2$ and let $A \in \mathscr{S} \mathscr{U} \mathscr{M}$ with $A^{k}=0$. Choose $I \triangle A$ minimal w.r.t. $0 \neq A / I \in \mathscr{M}$ and suppose $I \neq 0$. Choose $J \triangle I$ minimal w.r.t. $0 \neq I / J \in \mathscr{M}$. Since $A^{+}$is abelian, $A^{*} \cap J \triangle A$ (by Proposition 3.13). Let

$$
A^{\prime}=\frac{A}{A^{*} \cap J}, I^{\prime}=\frac{I}{A^{*} \cap J} \text { and } J^{\prime}=\frac{J}{A^{*} \cap J} \frac{J}{A^{*} \cap J} .
$$

Then $\quad J^{\prime} \triangle I^{\prime} \triangle A^{\prime}, \quad I^{\prime} / J^{\prime} \cong I / J \in \mathscr{M} \quad$ and $\quad A^{\prime} / I^{\prime} \cong A / I \in \mathscr{M}$ From Proposition 3.17, $\mathscr{R}\left(A^{\prime}\right) \subseteq \mathscr{R}\left(J^{\prime}\right)$ where $\mathscr{R}:=\mathscr{U} \mathscr{M}$. Since $\mathscr{S} \mathscr{R}=\mathscr{S} \mathscr{U} \mathscr{M}$ is hereditary, $\mathscr{R}\left(J^{\prime}\right)=\mathscr{R}\left(A^{\prime}\right)$. Using Proposition 3.14, we have $J^{k-1} \subseteq A^{k-1} \subseteq A^{*}$; hence $\left(J^{\prime}\right)^{k-1}=0$. Thus $\left(J^{\prime} / \mathscr{R}\left(J^{\prime}\right)\right)^{k-1}=0$. Moreover, from $J^{\prime} / \mathscr{R}\left(J^{\prime}\right) \in \mathscr{S} \mathscr{U} \mathscr{M}$ and the induction assumption, $J^{\prime} / \mathscr{R}\left(J^{\prime}\right) \in \mathscr{M}$. Consider the chain

$$
0 \triangle \frac{\mathscr{R}\left(J^{\prime}\right)}{\mathscr{R}\left(A^{\prime}\right)} \triangle \frac{J^{\prime}}{\mathscr{R}\left(A^{\prime}\right)} \triangle \frac{I^{\prime}}{\mathscr{R}\left(A^{\prime}\right)} \triangle \frac{A^{\prime}}{\mathscr{R}\left(A^{\prime}\right)}
$$

Using Proposition 3.15, we get $A^{\prime} / \mathscr{R}\left(A^{\prime}\right) \in \mathscr{M}$. Since $\mathscr{R}\left(A^{\prime}\right) \triangle A^{\prime}=A /\left(A^{*} \cap J\right), \mathscr{R}\left(A^{\prime}\right)$ is of the form $\mathscr{R}\left(A^{\prime}\right)=L /\left(A^{*} \cap J\right)$ where $L \triangle A$. This means $A / L \cong A^{\prime} / \mathscr{R}\left(A^{\prime}\right) \in \mathscr{M}$. But $L /\left(A^{*} \cap J\right)=\mathscr{R}\left(A^{\prime}\right)=\mathscr{R}\left(A^{\prime}\right) \subseteq J^{\prime}=J /\left(A^{*} \cap J\right)$ implies $L \subseteq J \subseteq I$. 
Our choice of $I$ implies $L=J=I$ which contradicts $0 \neq I / J$. Thus $I=0$ and $A \in \mathscr{M}$ which completes the proof.

Combining Proposition 3.18, Theorem 3.2 and Corollary 2.4 , we immediately have:

Theorem 3.19. Let $\mathscr{W}$ be an abelian and distributive $d_{3}(\mathcal{N})$-class. Then $\mathscr{M} \subseteq \mathscr{W}$ is a semisimple class if and only if $\mathscr{M}$ is regular, coinductive and closed under extensions.

\section{On the termination of the lower radical construction}

For all the (infinite) universal classes for which it is known that the lower radical construction terminates, it is either trivial and terminates at the second step (if the relation of being an ideal is transitive) or it has the first limit ordinal $\omega$ as upper bound (e.g. associative or alternative rings). The usual argument in showing that the lower radical construction terminates at $\omega$, is being able to prove the following for the generating class $\mathscr{M} \subseteq \mathscr{W}$ :

$$
\text { If } 0 \neq C \triangle \triangle A \text { and } C \in \mathscr{M}_{1} \text {, then } \bar{C} \in \mathscr{M}_{q} \text { for some finite } q
$$

where $\bar{C}$ is the ideal in $A$ generated by $C$.

We generalize this result and show that it in fact gives more than just the termination of the lower radical construction. Firstly, recall that an ordinal $\alpha$ is a $\gamma$-number (cf. Monk [12]) if it is 0 or of the form $\omega^{\beta}$ for some ordinal $\beta . \gamma$-numbers $\alpha$ has the (characterizing) property: $\varepsilon, \delta<\alpha$ implies $\varepsilon+\delta<\alpha$.

Theorem 4.1. Let $\mathscr{W}$ be a universal class of $\Omega$-groups such that for a fixed $\gamma$-number $\alpha$ $(\neq 0)$ and every chain $0 \neq J \triangle I \triangle A \in \mathscr{M}$, there is an ideal $B \triangle A$ with $0 \neq B \in\{J\}_{\beta}$ for some $\beta<\alpha(\beta$ depends on $J)$. Then every semisimple class in $\mathscr{W}$ is hereditary and the lower radical construction terminates at $\alpha$.

Proof. Let $\mathscr{M} \subseteq \mathscr{W}$ be a semisimple class, let $\mathscr{R}:=\mathscr{U} \mathscr{M}$ and consider the chain $\mathscr{R}(I) \triangle I \triangle A \in \mathscr{M}=\mathscr{S} \mathscr{R}$. If $\mathscr{R}(I) \neq 0$, our assumption on $\mathscr{W}$ yields an ideal $B \triangle A$ with $0 \neq B \in\{\mathscr{R}(I)\}_{\beta}$ for some $\beta<\alpha$. Since $\mathscr{R}(I) \in \mathscr{R}$, we have $B \in\{\mathscr{R}(I)\}_{\beta} \subseteq \mathscr{L}\{\mathscr{R}(I)\} \subseteq \mathscr{R}$ which contradicts $A \in \mathscr{S} \mathscr{R}$.

To prove the second assertion, let $\mathscr{M} \subseteq \mathscr{W}$ and let $B \in \mathscr{L} \mathscr{M}$. Let $A$ be any non-zero homomorphic image of $B$. Then $A \in \mathscr{L} \mathscr{M}$ and consequently it contains a non-zero accessible $\Omega$-subgroup $C$ with $C \in \mathscr{M}_{1}$. We complete the proof by showing that $A$ has a non-zero ideal $D$ which is in $M_{\sigma}$ for some $\sigma<\alpha$. Firstly, note that if $X \in \mathscr{M}_{\tau}$ for some $\tau$, then $\{X\}_{v} \subseteq \mathscr{M}_{\tau+v}$ for all ordinals $v$. Let us suppose that

$$
C=C_{1} \triangle C_{2} \triangle \cdots \triangle C_{n-1} \triangle C_{n}=A
$$

and we proceed by induction on $n$. 
For $n=3$, the assumption on $\mathscr{W}$ yields an ideal $D$ with $0 \neq D \in\{C\}_{\beta}$ for some $\beta<\alpha$. Then $D \in \mathscr{M}_{\sigma}$ where $\sigma=1+\beta<\alpha$.

Suppose the assertion holds for $n-1$. Then there is an ideal $E \triangle C_{n-1}$ with $0 \neq E \in \mathscr{M}_{v}$ for some $v<\alpha$. Consider the chain $0 \neq E \triangle C_{n-1} \triangle C_{n}=A$. By our assumption on $\mathscr{W}$, there is a non-zero ideal $D \triangle A$ with $D \in\{E\}_{\tau}$ for some $\tau<\alpha$. Then $D \in \mathscr{M}_{0}$ where $\sigma=\nu+\tau<\alpha$ which completes the proof.

Some of the properties considered in the first section can be expressed in terms of the lower radical determined by subideals. The proofs of the next two results are straightforward and are omitted. Also, whenever we have a chain $J \Delta I \triangle A \in \mathscr{W}, \bar{J}$ will denote the ideal in $A$ generated by $J$.

Proposition 4.2. The following are equivalent for any universal class $\mathscr{W}$ :

(1) For every chain $0 \neq J \triangle I \triangle A \in \mathscr{W}$, there is an ideal $B \triangle A$ with $0 \neq B \in \mathscr{L}\{J\}$.

(2) $\mathscr{S} \mathscr{L}\{A\}$ is hereditary for all $A \in \mathscr{W}$.

(3) Every semisimple class in $\mathscr{W}$ is hereditary.

Proposition 4.3. The following are equivalent for any universal class $\mathscr{W}$ :

(1) For every chain $0 \neq J \triangle I \triangle A \in \mathscr{W}, \bar{J} \in \mathscr{L}\{J\}$.

(2) $\mathscr{L}\{A\}$ has the ADS-property for all $A \in \mathscr{W}$.

(3) Every radical class in $\mathscr{W}$ has the ADS-property.

A special case of Theorem 4.1 is

Proposition 4.4. Let $\mathscr{W}$ be a universal class such that for every chain $0 \neq J \triangle I \triangle A \in \mathscr{W}$ there is a finite number $q$ (depending on $J$ ) such that $\bar{J} \in\{J\}_{q}$. Then the lower radical construction in $\mathscr{W}$ terminates at $\omega$.

Theorem 4.5.* Let $\mathscr{W}$ be a $d_{4}$-class. Then, for every chain $0 \neq J \Delta I \triangle A \in \mathscr{W}, \bar{J} \in\{J\}_{2}$ holds (or equivalently, every non-zero homomorphic image of $\bar{J}$ contains a non-zero ideal which is a homomorphic image of $J$ ). Hence the lower radical construction terminates at $\omega$.

For alternative rings, this result has been proved by Krempa [8]. Since his proof only uses the isomorphism theorems (his Lemma 3 is provided by our assumption on $\mathscr{W}$ ), the proof carries over verbatim.

Quite often, for certain classes $\mathscr{M}$, the lower radical construction terminates at the second or third step. We give a few examples.

*E. R. Puczylowski (On general theory of radicals) has proved that the lower radical construction terminates at $\omega$ in a $d_{3}(\mathscr{B})$-class. 
Proposition 4.6. Let $\mathscr{W}$ be a $d_{2}(\mathscr{A})$-class or a $d_{1}(\mathscr{A})$-class with $\mathscr{A}$ hereditary. If $\mathscr{M} \subseteq \mathscr{U} \mathscr{A}$ (or equivalently, if $\mathscr{M}_{1} \cap \mathscr{A}=0$ ), then $\mathscr{L} \mathscr{M}=\mathscr{M}_{2}$.

Proof. Let $A \in \mathscr{M}_{3}$ and let $B$ be any non-zero homomorphic image of $A$. Since $B \in \mathscr{M}_{3}$, there is a chain $0 \neq J \triangle I \triangle B$ with $J \in \mathscr{M}_{1} \subseteq \mathscr{U} \mathscr{A}$. From Proposition $2.9, J \triangle A$ holds and we are done.

Apart from the properties of the $d_{i}$-classes in the general radical theory of associative rings, another important property is the validity of the Andrunakievič Lemma. In order to give more examples of classes for which the lower radical construction terminates at the second or third step, we introduce a condition which generalizes the Andrunakievič Lemma: $\mathscr{W}$ satisfies condition $a(\mathscr{A})$ if for any chain $0 \neq J \triangle I \triangle A \in \mathscr{W}$ there are ideals $B_{1}, B_{2}$ in $A$, not both zero, with $B_{1} \subseteq J, B_{2} \subseteq I$ and if $B_{1}=0$, then $B_{2} \in \mathscr{A}$.

Proposition 4.7. Let $\mathscr{W}$ satisfy condition a(A). If $\mathscr{M} \subseteq \mathscr{W}$ is hereditary and homomorphically closed with $\mathscr{A} \subseteq \mathscr{M}$, then $\mathscr{L} \mathscr{M}=\mathscr{M}_{2}$.

Proof. Let $B \in \mathscr{M}_{3}$ and let $A$ be any non-zero homomorphic image of $B$. Since $B \in \mathscr{M}_{3}$, there is a chain $0 \neq J \triangle I \triangle A$ with $J \in \mathscr{M}_{1}=\mathscr{M}$. By our assumption on $\mathscr{W}$, there are ideals $B_{1}, B_{2} \triangle A$ with $B_{1} \subseteq J$ and $B_{2} \subseteq I$. If $B_{1} \neq 0$, then $B_{1} \in \mathscr{M}_{1}$ by the hereditariness of $\mathscr{M}_{1}$; if $B_{1}=0$, then $0 \neq B_{2} \in \mathscr{A} \subseteq \mathscr{M}=\mathscr{M}_{1}$. Hence $B \in \mathscr{M}_{2}$.

Proposition 4.8. Let $\mathscr{W}$ be a $d_{4}(\mathscr{B})$-class which satisfies condition a $(\mathscr{B})(\mathscr{B}$ is as defined in Section 3). Then $\mathscr{L} \mathscr{M}=\mathscr{M}_{3}$ for any hereditary and homomorphically closed subclass $\mathscr{M} \subseteq \mathscr{W}$.

Proof. Let $B \in \mathscr{M}_{4}$ and let $A$ be any non-zero homomorphic image of $B$. Since $A \in \mathscr{M}_{4}$, there is a chain $0 \neq I_{1} \Delta I_{2} \Delta I_{3} \Delta I_{4}=A$ with $I_{i} \in \mathscr{M}_{i}$ for $i=1,2,3,4$. Considering $I_{2} \triangle I_{3} \triangle I_{4}=A$ and applying condition $a(\mathscr{B})$, we have two ideals $B_{1}$ and $B_{2}$ of $A$, not both zero with $B_{1} \subseteq I_{2}$ and $B_{2} \subseteq I_{3}$. Since $\mathscr{M}=\mathscr{M}_{1}$ is hereditary, so is $\mathscr{M}_{k}$ for all $k$ and we have $B_{1} \in \mathscr{M}_{2}$ and $B_{2} \in \mathscr{M}_{3}$. If $B_{1} \neq 0$, we are done. Suppose thus $B_{1}=0$. Then $0 \neq B_{2} \in \mathscr{B}$. Since $B_{2} \in \mathscr{M}_{3}$, there are ideals $0 \neq X \triangle Y \triangle B_{2}$ with $X \in \mathscr{M}_{1}$. But $B_{2} \in \mathscr{B}$; hence $X \triangle B_{2}$. Then $0 \neq \bar{X} \in\{X\}_{2} \subseteq \mathscr{M}_{2}$ (where $\bar{X}$ is the ideal in $A$ generated by $X$ ) from Theorem 4.5. Thus $A \in \mathscr{M}_{3}$, which proves the assertion.

\section{Examples}

5.1. Any universal class $\mathscr{W}$ of groups is a $d_{4}$-class. Indeed, if $J \Delta I \triangle A$ and $J \Delta A$, then there is an $a \in A$ such that $a+J-a \notin J$. Let $C:=(a+J-a)+J$. Then $C \triangle I$ and $\delta: J \rightarrow C / J$ defined by $\delta(x)=(a+x-a)+J$ is the desired homomorphism. It is not known whether Sands' Theorem is valid for groups.

5.2. It is well-known that any universal class of associative or alternative rings is a 
$d_{4}(\mathscr{Z})$-class $\left(=d_{4}(\mathscr{T})\right.$-class), and thus a $d_{4}(\mathscr{B})$-class-cf. Anderson, Divinsky and Suliński [1], Terlikowska-Oslowska $[18,19]$ and Krempa [8].

5.3. Let $\mathscr{W}$ be any universal class of $\Gamma$-rings. Then $\mathscr{W}$ is a $d_{4}(\mathscr{B})$-class. This can easily be verified as for the associative ring case.

5.4. In this example we consider a variant on the cubic rings introduced by Nobusawa [13]. An abelian group $M$ is a left commutative and associative cubic ring if it is endowed with a ternary operation such that for all $a, b, c, d, e \in M$ the following holds:

(1) $(a+b) c d=a c d+b c d$

(2) $a(b+c) d=a b d+a c d$

(3) $a b(c+d)=a b c+a b d$

(4) $a b(c d e)=(a b c) d e=a(b c d) e$ (associativity)

(5) $a b c=b a c$ (left commutativity).

Then $A \triangle M$ if and only if $A$ is a subgroup of $M, M M A \subseteq A$ and $M A M \subseteq A$. Any universal class of left commutative associative cubic rings is a $d_{4}(\mathscr{B})$-class (as can easily be verified as for the associative ring case).

5.5. A non-associative ring $A$ is an autodistributive ring if $a(b c)=(a b)(a c)$ and $(a b) c=(a c)(b c)$ for all $a, b, c \in A$. Let $\mathscr{W}$ be a universal class of autodistributive rings. Then $\mathscr{W}$ is a $d_{4}(\mathscr{B})$-class. Indeed, if $J \triangle I \triangle A \in \mathscr{W}$ and $J \not A$, then here is an $a \in A$ such that $J a \notin J$ or $a J \notin J$. Suppose $J a \nsubseteq J$ (the other case is treated similarly). Let $C=J a+J$. Then $C \triangle I$ and $\delta: J \rightarrow C / J$ defined by $\delta(x)=x a+J$ is the required homomorphism with $C / J \in \mathscr{B} \backslash\{0\} . \mathscr{W}$ satisfies condition $a(\mathscr{B})$ - this follows from Corollary 2.7 and Proposition 2.8 in Anderson and Gardner [2].

5.6. Let $\mathscr{V}$ be the variety of all distributive near-rings (or equivalently, all non-abelian rings). Any such near-ring $N$ has the property that $N^{2}$ is abelian, i.e. $a b+c d=c d+a b$ for all $a, b, c, d \in N$ (cf. Pilz [14]). Although this variety satisfies $\bar{J}^{3} \subseteq J$ for all $J \triangle I \triangle A \in \mathscr{V}$ where $\vec{J}^{3}:=\{a b c \mid a, b, c \in \bar{J}\}$ (note that the powers defined for subsets of near-rings is not in harmony with the $\Omega$-group theoretic notion-cf. Section 3 ), it is not known what results (if any) from Section 2 and Section 3 are valid in $\mathscr{r}$. Motivated by the next result, we can improve matters if we have some substitute for the lack of abelianness.

Proposition 5.6.1. Let $\mathscr{R} \subseteq \mathscr{V}$ be a radical class. Then $\mathscr{S} \mathscr{R}$ is hereditary if and only if both $a \mathscr{R}(I)$ and $\mathscr{R}(I) a$ are normal subgroups of $A$ for all $a \in A$ and $I \triangle A \in \mathscr{S} \mathscr{R}$.

Proof. Suppose $\mathscr{S} \mathscr{R}$ is hereditary and let $I \triangle A \in \mathscr{S} \mathscr{R}$ and $a \in A$. Then $\mathscr{R}(I)=0$ and both $a \mathscr{R}(A)$ and $\mathscr{R}(I) a$ normal subgroups of $A$ follows trivially. Conversely, let $I \triangle A \in \mathscr{S} \mathscr{R}$. If $\mathscr{R}(I) \not A$, then $\mathscr{R}(I)$ is not normal in $A$ or $\mathscr{R}(I)$ is normal in $A$ but there 
is an $a \in A$ such that $a \mathscr{R}(I) \nsubseteq \mathscr{R}(I)$ or $\mathscr{R}(I) a \ddagger \mathscr{R}(I)$ (without loss of generality, we may only assume $a \mathscr{R}(I) \nsubseteq \mathscr{R}(I)$ ). Let $J=\mathscr{R}(I)$ and suppose it is not normal in $A$. Then there is an $a \in A$ such that $a+J-a \notin J$. Then $C$, defined by $C=(a+J-a)+J$, is normal in $I$ and since $A^{2}$ is abelian, it is an ideal in $I$. Define a mapping $\phi: J \rightarrow C / J$ by $\phi(x)=(a+x-a)+J$. Clearly $\phi$ is surjective, preserves addition and $\phi(x) \phi(y)=0$. Moreover, since $x \mathscr{R}(I)$ is normal in $A$, also $\phi(x y)=(a+x y-a)+J=0$. Hence $C / J$ is a nonzero homomorphic image of $J=\mathscr{R}(I) \in \mathscr{R}$. But this contradicts $C / J \triangle I / J \in \mathscr{S} \mathscr{R}$. Suppose thus that $J$ is normal in $A$ but $a J \nsubseteq J$ for some $a \in A$. Let $D$ be defined by $D=a J+J$. Then $D \Delta I$ and the mapping $\phi: J \rightarrow D / J$ defined by $\phi(x)=a x+J$ is a surjective homomorphism. As above, $D / J \in \mathscr{R}$ and $0 \neq D / J \triangle I / J \in \mathscr{S} \mathscr{R}$, which is impossible. Hence $\mathscr{R}(I)=J \triangle A \in \mathscr{S} \mathscr{R}$ and $I \in \mathscr{S} \mathscr{R}$ follows.

Corollary 5.6.2. Let $\mathscr{R} \subseteq \mathscr{V}$ be a radical class. Then $\mathscr{S} \mathscr{R}$ is hereditary if and only if $a \mathscr{R}(I)=0=\mathscr{R}(I)$ a for all $I \triangle A \in \mathscr{S} \mathscr{R}$ and $a \in A$.

Let us now fix some notation. If $K$ is a normal subgroup of $A \in \mathscr{V}$, it will be donated by $K \Delta A$ and the normal subgroup of $A$ generated by $a \in A$ will be denoted by [a].

Proposition 5.6.3. Let $A$ be a distributive near-ring. Then the following are equivalent:

(a) $a K \Delta A$ for all $K \Delta A, a \in A$.

( $\beta$ ) For all $x, y, z \in A$, there exists an integer $n$ (depending on $x, y$ and $z$ ) such that $z+x y-z=n(x y)$.

(y) $K a \Delta A$ for all $K \Delta A, a \in A$.

Proof. If $(\alpha)$ is assumed, let $x, y, z \in A$. Then $x[y] \Delta A$ and $z+x y-z=x c$ for some $c \in[y]$. Since $c$ is of the form

$$
c=\sum_{i=1}^{m}\left(d_{i}+n_{i} y-d_{i}\right)
$$

with $d_{i} \in A$ and each $n_{i}$ some integer, we have

$$
\begin{aligned}
z+x y-z & =\sum_{i=1}^{m} n_{i}(x y) \quad \text { since } A^{2} \text { abelian } \\
& =n(x y) \quad \text { where } n=\sum_{i=1}^{m} n_{i} .
\end{aligned}
$$

Hence $(\beta)$ holds. The other equivalences can also easily be verified.

Classes of near-rings which satisfy condition $(\alpha)$ have recently been considered by Anderson, Kaarli and Wiegandt [3] in connection with left strong radicals of near-rings. 
In the sequel, let $\mathscr{W}$ be a universal class of distributive near-rings which satisfies condition $(\alpha)$. Easy examples show that $\mathscr{W} \varsubsetneqq \mathscr{V}$ and there are distributive near-rings that satisfy condition $(\alpha)$, but need not be a ring (as the zero multiplication on any nonabelian group can testify). Using the same type of arguments as in the proof of Proposition 5.6.1, it can be verified that $\mathscr{W}$ is a $d_{4}(\mathscr{Z})$-class where $\mathscr{Z}:=\{A \in \mathscr{W} \mid a b=0$ for all $a, b \in A\}$.

Since the underlying groups in $\mathscr{W}$ need not be abelian, the nearest we can get to a version of Sands' Theorem in $\mathscr{W}$ is: $\mathscr{M} \subseteq \mathscr{W}$ is a semisimple class iff $\mathscr{M}$ is regular, coinductive, closed under extensions and if $I \triangle A \in \mathscr{M}$ and $I^{2}=0$, then $I \in \mathscr{M}$. We do not know if the last condition imposed on $\mathscr{M}$ is really necessary.

\section{REFERENCES}

1. T. Anderson, N. Divinsky and A. Sulinsskı, Hereditary radicals in associative and alternative rings, Canad. J. Math. 17 (1965), 594-603.

2. T. Anderson and B. J. Gardner, Semi-simple classes in a variety satisfying an Andrunakievič Lemma, Bull, Austral. Math. Soc. 18 (1978), 187-200.

3. T. Anderson, K. KaArli and R. Wiegand, On left strong radicals of near-rings, Proc. Edinburgh Math. Soc. 31 (1988), 447-456.

4. T. Anderson and R. Wiegandt, Semisimple classes of alternative rings, Proc. Edinburgh Math. Soc. 25 (1982), 21-26.

5. P. N. ÁNH, N. V. Lol and R. WIEGANDT, On the radical theory of Andrunakievič varieties, Bull. Austral. Math. Soc. 31 (1985), 257-269.

6. P. N. ÁNH and R. WIEGANDT, Semisimple classes of non-associative rings and Jordan algebras, Comm. Algebra 13 (1985), 2669-2690.

7. P. J. Higgins, Groups with multiple operators, Proc. London Math. Soc. 6 (1956), 366-416.

8. J. Krempa, Lower radical properties for alternative rings, Bull. Acad. Polon. Sci. 23 (1975), $139-142$.

9. A. Kurosh, Radicals of rings and algebras, Math. Sbornik 33 (1953), 13-26 (in Russian. English translation in: Coll. Math. J. Bolyai 6. Rings, modules and radicals, North-Holland, 1973, 297-312).

10. W. G. Leavitt and E. P. Armendariz, Nonhereditary semisimple classes, Proc. Amer. Math. Soc. 18 (1967), 1114-1117.

11. N. V. Lot and R. Wiegandt, Involution algebras and the Anderson-Divinsky-Sulinski property, Acta Sci. Math. Szeged 50 (1986), 5-14.

12. J. D. Monk, Introduction to Set Theory (McGraw-Hill Inc., USA, 1969).

13. N. Nobusawa, On a generalization of the ring theory, Osaka J. Math. 1 (1964), 81-89.

14. G. PILz, Near-rings (North-Holland/American Elsevier, Amsterdam, 1977).

15. E. R. Puczylowskı, On semisimple classes of associative and alternative rings, Proc. Edinburgh Math. Soc. 27 (1984), 1-5.

16. A. D. SAnds, A characterization of semisimple classes, Proc. Edinburgh Math. Soc. 24 (1981), 5-7. 
17. A. Suliński, T. Anderson and N. Divinsky, Lower radical properties for associative and alternative rings, J. London Math. Soc. 41 (1966), 417-424.

18. B. Terlikowska-Oslowska, Category with a self-dual set of axioms, Bull. Acad. Polon. Sci. 25 (1977), $1207-1214$.

19. B. Terlikowska-Oslowska, Radical and semisimple classes of objects in categories with a self-dual set of axioms, Bull. Acad. Polon. Sci. 26 (1978), 7-13.

20. L. C. A. van Leeuwen and R. Wiegandt, Radicals, semisimple classes and torsion theories, Acta Math. Acad. Sci. Hungar. 36 (1980), 37-47.

21. L. C. A. van Leeuwen and R. Wiegand, Semisimple and torsionfree classes, Acta Math. Acad. Sci. Hungar. 38 (1981), 73-81.

22. S. VeldSMAN, Modulo-constant ideal-hereditary radicals of near-rings, Quaestiones Math. 11 (1988), 253-278.

Department of Mathematics

University of Port Elizabeth

P.O. Box 1600

6000 Port Elizabeth

SOUTH Africa 